\title{
Empowerment del alumnado para la selección de actividades y espacios para la dinámica de los baños de realidad
}

 \\ Esgueva López ${ }^{\mathfrak{c}}$ y María de-Miguel-Molina ${ }^{\mathrm{d}}$ \\ aUniversitat Politècnica de València, macamon@crbc.upv.es, 'buniversitat Politècnica de València, \\ virsanca@crbc.upv.es 'Universitat Politècnica de València, maeslo2@dib.upv.es y ${ }^{d}$ Universitat \\ Politècnica de València, mademi@omp.upv.es
}

\begin{abstract}
This proposal aims to demonstrate the effectiveness of the implementation of empowerment in the university classroom. Currently, students demand greater prominence within their own learning. The present methodological project, is born from the detection of the need to give voice to the students, obviously from a very studied perspective, consensual and conducted by the teaching staff, so that they can make proposals that can be materialized, thus feeling part of their own educational process. In this case we will expose the use of the aforementioned methodological tool for the selection of the Reality Baths activities developed in subjects of the Degree in Conservation and Restoration of Cultural Assets.
\end{abstract}

Keywords: empowerment, reality baths, teaching innovation, teaching outside the classroom, company, work reality, integration

\begin{abstract}
Resumen
Esta propuesta pretende demostrar la eficacia de la implementación del empowerment en el aula universitaria. En la actualidad, el alumnado demanda mayor protagonismo dentro de su propio aprendizaje. El presente proyecto metodológico, nace de la detección de la necesidad de dar voz al alumnado, obviamente desde una perspectiva muy estudiada, consensuada y conducida por el profesorado, de modo que puedan realizar propuestas que se puedan llegar a materializar, sintiéndose así parte de su propio proceso educativo. En este caso expondremos el uso de la citada herramienta metodológica para la selección de las actividades y espacios de baños de realidad desarrolladas en asignaturas del Grado en Conservación y Restauración de Bienes Culturales.
\end{abstract}

Palabras clave: empoderamiento, baños de realidad, innovación docente, docencia fuera de las aulas, empresas, realidad laboral, integración, 


\section{Introducción}

El presente artículo se presenta en el marco del Equipo de Innovación y Calidad Educativa EICE “AFA-Realidad El proceso de Enseñanza y Aprendizaje Fuera de las Aulas - Baños de Realidad”, del Instituto de Ciencias de la Educación de la Universitat Politècnica de València. Dentro de las dinámicas de búsquedas de espacios docentes fuera de las aulas de la universidad, se emplea la herramienta de baños de realidad desde el curso 2016-2017, con el Proyecto de Innovación y Mejora Educativa (PIME) denominado "El proceso de Enseñanza y Aprendizaje Fuera de las Aulas de la Facultad de Bellas Artes: Baños de Realidad”.

Con los citados baños de realidad se pretende transferir el conocimiento científico de la disciplina al alumnado, a partir del planteamiento tutorizado de la diversidad profesional con la que se enfrentará al acabar sus estudios (Carabal et al, 2018). En este caso se pretende ahondar en la comparativa de los resultados obtenidos de esta innovación educativa en la asignatura de Introducción a la Conservación y Restauración de Dorados y Policromías (cód.14068) del Grado en Conservación y Restauración de Bienes Culturales de la UPV. En este caso, los baños de realidad han sido consensuados con el alumnado, empleando para ello la metodología inclusiva de empowerment del alumnado, que ha servido para generar una puesta en común de sus inquietudes, para acabar determinando el espacio donde efectuar el baño de realidad y las actividades a realizar.

El empowerment pretende, como principal objetivo, "el empoderamiento social entre los universitarios españoles, y su percepción de la universidad como facilitador en la educación en el uso de los medios para convertirse en ciudadanía activa y crítica" (Gavilán et al., 2017). Para ello, con la presente práctica, se ofrecen las herramientas pertinentes y se propone un acercamiento a esta técnica desde las aulas universitarias.

La innovación baños de realidad, dentro del ámbito universitario, busca que la formación presencial no esté sujeta únicamente al espacio universitario, implementando nuevos espacios formativos, siguiendo una estrategia conjunta. De este modo se ha implicado al alumnado en la selección de espacios laborales reales, como instituciones públicas o empresas, involucrándoles en la realidad diaria de los procesos científicos de la conservación y la restauración de bienes culturales.

Con el presente artículo se analizan los resultados obtenidos al fomentar el uso de estrategias de docencia alternativa basadas en metodologías activas, mediante una inmersión social tecnológica y cultural que potencia el aprendizaje continuo. Para esta tipología de innovación docente se ha hecho especial hincapié en el trabajo en grupo y en el contacto con casos reales en los que se encontrará el alumnado en su futuro laboral.

Partimos de la base de que la motivación es el motor del aprendizaje (Ospina Rodríguez, 2006). Con esta práctica se propone testar el alcance de la misma, teniendo en cuenta la motivación que puede generar en el alumnado, al tener en cuenta sus inquietudes e intereses específicos, que en la inmensa mayoría de los casos van enfocados a su posterior ámbito laboral, haciendo que potencien el pensamiento crítico, valorando las necesidades reales con las que se encontrarán al acabar sus estudios. 
La puesta en marcha de las actividades propuestas en las asignaturas implicadas en este proyecto, fomentan la adquisición de las siguientes competencias transversales: Comprensión e integración, Innovación, creatividad y emprendimiento, Diseño y proyecto, Comunicación efectiva y Conocimiento de problemas contemporáneos.

Tras una búsqueda exhaustiva en revistas científicas y bases de datos, podríamos afirmar que no existe una extensa bibliografía específica al respecto del método propuesto, puesto que se trata de una innovación de la cual no hay evidencias de ejemplos prácticos de su testado en otras instituciones. El autor Analí Torres realiza un análisis teórico del empleo de empoderamiento como una de las piezas claves para una "perspectiva emancipadora del acto pedagógico, que se sustenta en el empoderamiento del estudiante como una vía para la transformación individual y social. Desde esta perspectiva el empoderamiento es concebido como el proceso de concienciación que da cuenta al estudiante de sus capacidades y potencialidades y la relación de éstas con el mundo que lo rodea" (Torres, 2009). Respecto al empowerment docente se han encontrado casos de aplicación internacional (Terán Cazares, 2015) (García de Pilo et al, 2014), pero aplicado a los docentes, para que perciban la posibilidad de ser partícipes en las políticas de sus programas educativos. Pero en este caso no se aplica a los docentes, sino al alumnado, con lo cual es una práctica innovadora el transferir esa potente herramienta al mismo.

\section{Objetivos}

\section{Objetivo general}

$1^{\circ}$ Demostrar la eficacia del uso del empowerment durante la impartición del grado, para que los alumnos ayuden a seleccionar los espacios en los vean aplicada la teoría y la práctica desarrollada en el aula, a espacios y propuestas de casos teóricos y prácticos en el ámbito real. Asimismo, por medio de la misma herramienta, otorgar la posibilidad de seleccionar las actividades a realizar, para que éstas respondan a sus expectativas. El ámbito de aplicación ha sido en la dinámica de baños de realidad.

\section{Objetivos específicos}

$2^{\circ}$ Hacer participes a los alumnos de propuestas docentes, con casos con los que podrán enfrentarse en su futura realidad laboral, relacionándolos con la teoría y práctica desarrollada en el aula, trabajando sus habilidades relacionadas con la autonomía y la profesionalización.

$3^{\circ}$ Reflexionar acerca de la necesidad de implementar actividades inclusivas, que tengan en cuenta la opinión del alumnado, y de las ventajas de que los alumnos se sientan protagonistas de su propio aprendizaje, como estrategia para la integración de contenidos.

$4^{\circ}$ Aportar a los alumnos una visión analítica de los contenidos trabajados, para que puedan desarrollar su pensamiento práctico y crítico en ámbitos reales, promoviendo la comunicación eficiente.

$5^{\circ}$ Promover el trabajo en equipo, el desarrollo de metodologías cooperativas, liderazgo y la resolución de problemas. 
$6^{0}$ Potenciar la igualdad de condiciones y la paridad en las propuestas y solicitudes relacionadas con la actividad.

$7^{\circ}$ Transferir los resultados de dicha estrategia didáctica a la comunidad universitaria.

\section{Desarrollo de la innovación}

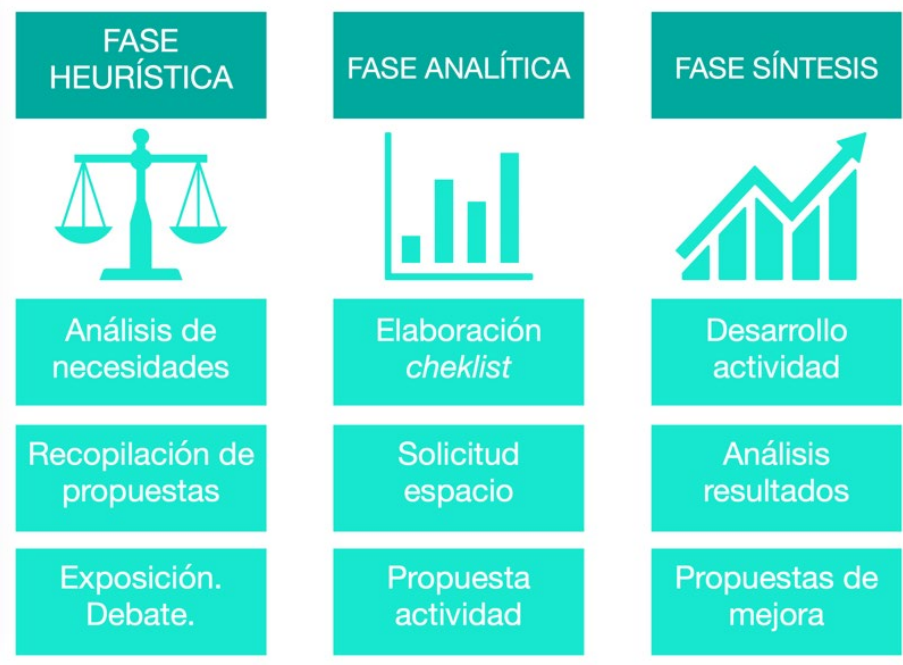

Figura 1. Esquema de la metodología llevada a cabo. Fuente: elaboración propia.

\section{FASE HEURÍSTICA}

1. En primer lugar se realizó un análisis de las necesidades específicas a cubrir en la asignatura Introducción a la Conservación y Restauración de Dorados y Policromías, relacionadas con la segunda parte del temario, vinculada a la conservación y restauración. Con base en los contenidos que se pretendieron reforzar, se señalaron una serie de espacios en los que efectuar los baños de realidad. Éstos basan su esencia en extrapolar contenidos vistos y tratados en clase a espacios reales en los que se trabajan dichos contenidos de modo real. Con ello, la experiencia es práctica y en ella se trabajan contenidos sin efectuar intervenciones sobre la materia directamente, pero sí se presentan propuestas, dudas y se generan espacios y situaciones reales con los que un conservador-restaurador de bienes culturales se puede encontrar en su futuro laboral.

Ante el amplio escenario laboral que puede presentarse en el futuro del alumnado en esta disciplina, es necesario ahondar previamente en qué espacios laborales pretenden ser estudiados en profundidad.

2. Recopilación de las propuestas del alumnado, durante la docencia presencial y no presencial, mediante guiones establecidos con anterioridad. Con ello, el alumnado, ante la selección del espacio donde efectuar las prácticas, realizó una evaluación por medio de la herramienta SuveyMonkey®. 
Los resultados obtenidos respecto a los espacios en los que efectuar la práctica (Figura 2) fueron museos en un $84 \%$, instituciones públicas $66 \%$, empresa-autónomo un $63 \%$, galerías $43 \%$, otros espacios expositivos un 5\%. Entre el 5\% final, dedicado a "otros", que permite la posibilidad de escribir libremente respuestas abiertas, el alumnado expone reiterativamente la idea principal: "Tenemos un ámbito de actuación muy amplio como conservadores restauradores y cualquier espacio expositivo o de trabajo es adecuado para realizar prácticas siempre que estén bien enfocadas".



Figura 2. Encuesta con SurveyMonkey®. Fuente: elaboración propia.

Asimismo, la citada encuesta no solo se centraba en el espacio formativo a nivel temático, sino también de modo concreto, relacionado con actividades que quisiesen realizar y con espacios reales de la ciudad de València.

Respecto a las actividades a realizar, el porcentaje máximo era el relacionado con la conservación-restauración de bienes culturales (Figura 3), con un 91\%, las dedicadas a estudio de los bienes culturales con un $51 \%$, los que su finalidad es la gestión cultural, con un $46 \%$, y posteriormente con comercialización y/o exposición de obras de arte (37\%), y la realización de obras $(21 \%)$.

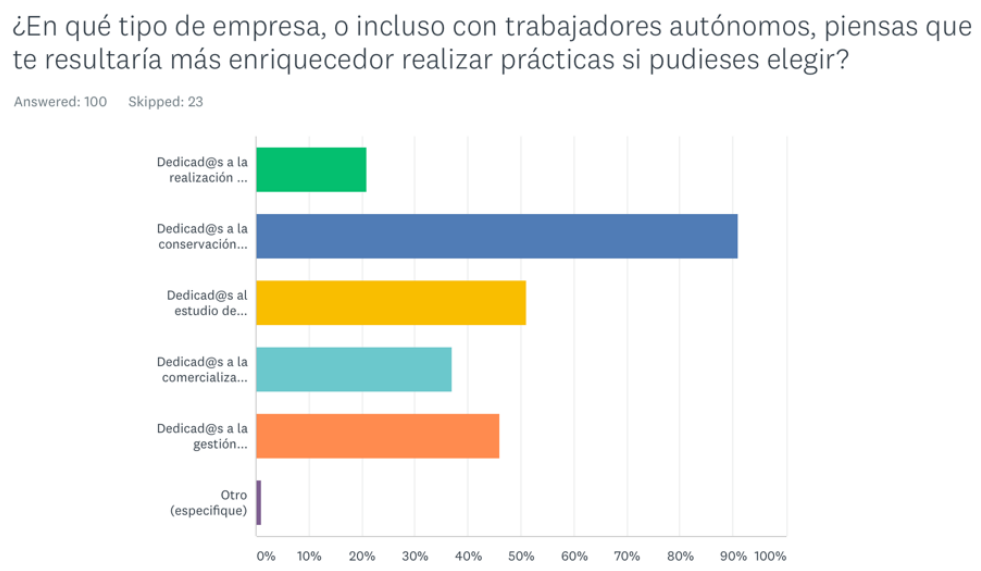

Figura 3. Encuesta con SurveyMonkey®. Fuente: elaboración propia. 
En última instancia, las respuestas relacionadas con los espacios concretos (Figura 4) dieron como resultado la realización del baño de realidad en Institut Valencià de Conservació i Restauració de Béns Culturals (IVC+R) con un $83 \%$ de los votos, seguido por el Museo de Bellas Artes de Valencia (San Pío V) con un 62\%, tras él el Institut Valencià d'Art Modern (IVAM). Centre Julio González, con un 59\%. Tras ellos, el Museo Nacional de Cerámica y Artes Suntuarias Gonzalez Martí (36\%), Museo Valenciano de la Ilustración y la Modernidad (MuVIM), con un 35\%, Museo Valencià d'Etnologia (33\%), Museo y Colegio del Arte Mayor de la Seda (26\%) y el Museo Fallero con un 23\%.
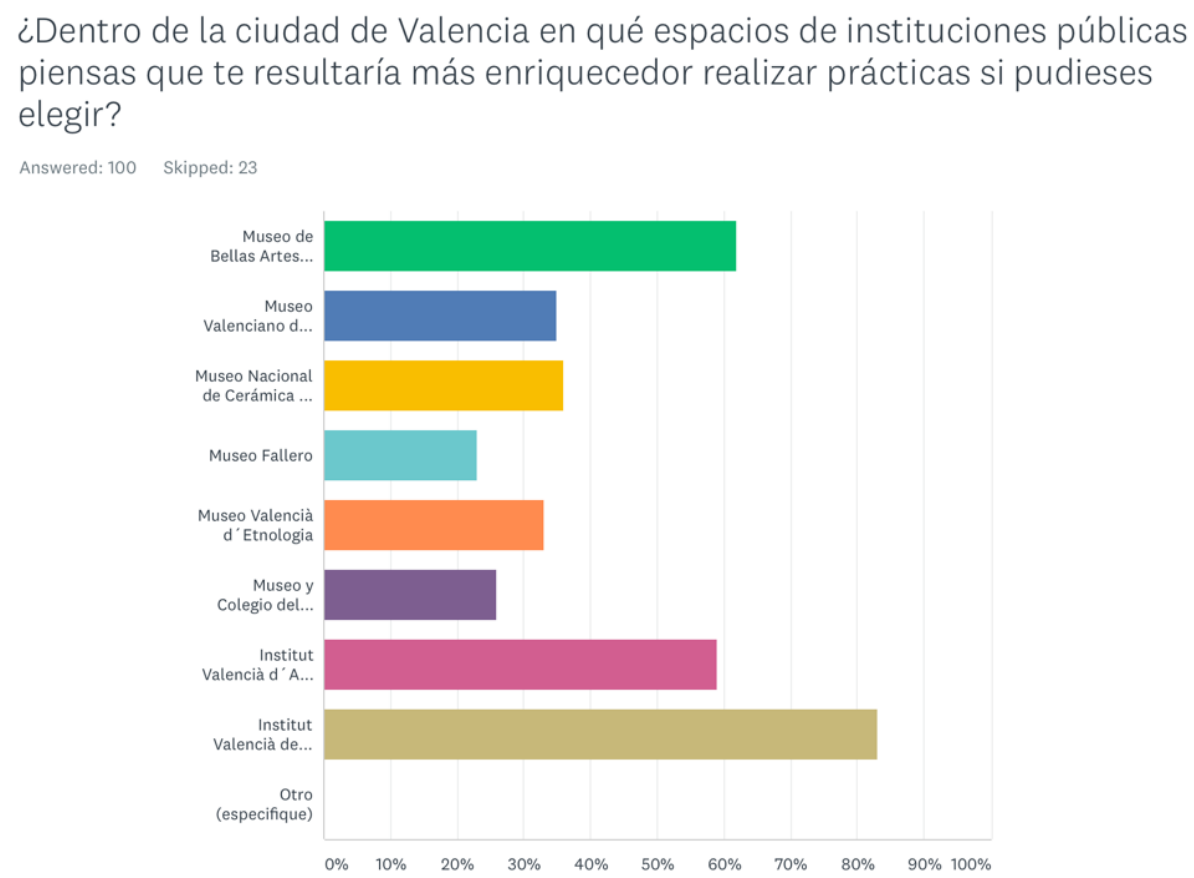

Figura 4. Encuesta con SurveyMonkey®. Fuente: elaboración propia.

Asimismo se realizó un sondeo entre el alumnado referente a las actividades a realizar, en los que se mostraron las expectativas de la práctica fuera del aula (Figura 5). 


\section{En caso de realización de prácticas fuera del aula ¿qué actividad sería más acorde con tus expectativas?}

Answered: 100 Skipped: 23

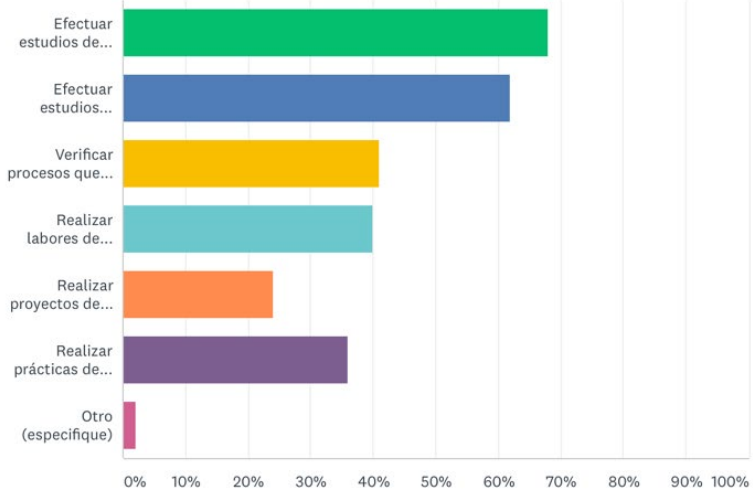

\begin{tabular}{|c|c|c|c|}
\hline ANSWER CHOICES & - & RESPON & ES - \\
\hline - Efectuar estudios de naturaleza de las obras, materiales, técnicas de manufactura, etc. & & $68.00 \%$ & 68 \\
\hline - Efectuar estudios ambientales y de definición de estado de conservación de las obras & & $62.00 \%$ & 62 \\
\hline - Verificar procesos que se efectúan en la realidad laboral mediante la observación paralela a la actividad & & $41.00 \%$ & 41 \\
\hline - Realizar labores de gestión expositiva y cultural & & $40.00 \%$ & 40 \\
\hline - Realizar proyectos de diseño y maquetación & & $24.00 \%$ & 24 \\
\hline - Realizar prácticas de acompañamiento en recorridos expositivos & & $36.00 \%$ & 36 \\
\hline - Otro (especifique) & Responses & $2.00 \%$ & 2 \\
\hline
\end{tabular}

Figura 5. Encuesta con SurveyMonkey®. Fuente: elaboración propia.

Con ello los resultados fueron, con un $68 \%$, efectuar estudios de naturaleza de las obras, materiales, técnicas de manufactura, etc. El siguiente fue relacionado con los estudios ambientales y de definición del estado de conservación de las obras, con un $62 \%$. La verificación de procesos de la realidad laboral mediante la observación paralela a la actividad fue valorada con un $41 \%$. Realizar labores de gestión expositiva y cultural fue votado por un $40 \%$ del alumnado. Efectuar prácticas de acompañamiento en recorridos expositivos fue valorado con un $36 \%$ y la realización de proyectos de diseño y maquetación fue valorada con un $24 \%$.

3. División del alumnado por grupos teniendo en cuenta sus selecciones. Sesión de exposición de los motivos de la selección de las propuestas por parte de cada grupo de trabajo y posterior debate, en las que se plantearon las posibilidades de cada una de ellas. Según Esteban García y Ortega Gutiérrez "La utilización del debate puede ser una estrategia muy útil para el desarrollo intelectual del estudiante y para conectar los temas que se tratan en la universidad con los problemas sociales" (Esteban et al, 2017). En esta primera fase, el profesorado mediante la observación, realizó un barrido de las opiniones de los diferentes equipos, teniendo en cuenta también la valoración entre grupos de las propuestas presentadas.

\section{FASE ANALÍTICA}

1. Elaboración de un Checklist para ayudar al profesorado a establecer unas pautas de viabilidad con respecto a los espacios y las actividades seleccionadas. En él, de manera 
cualitativa, se evalúan las posibilidades potenciales que presenta cada uno de los espacios seleccionados y se introduce la valoración del profesorado respecto a cada una de las mismas, relacionándolas con las actividades que se proponen.

2. Se realizan las pertinentes gestiones, por parte del profesorado, para poder llevar a cabo la actividad docente en el espacio seleccionado. Es importante realizarlo con el tiempo necesario para que sea factible. Si no fuese posible, se trataría de realizar en el siguiente espacio seleccionado en las encuestas efectuadas.

3. Propuesta de la nueva actividad seleccionada. Adaptación a la realidad docente, en caso necesario, de las actividades que planteen, teniendo en cuenta que no se va a realizar una práctica de empresa, sino que se va a efectuar un baño de realidad, que como hemos comentado con anterioridad, plantea al alumnado un cambio de rol, presentándole actividades para que experimente una inmersión laboral, pero sin realizar práctica alguna en la materia.

\section{FASE SÍNTESIS}

1. Desarrollo de la actividad. Implementación del baño de realidad durante la docencia presencial, en el espacio seleccionado.

2. Evaluación de resultados. Se valorará la opinión del profesorado y la del alumnado. El profesorado calificará la práctica, teniendo en cuenta las competencias y competencias transversales, fundamentalmente, que se han trabajado en la práctica, que como se ha expuesto con anterioridad son: Comprensión e integración, Innovación, creatividad y emprendimiento, Diseño y proyecto, Comunicación efectiva y Conocimiento de problemas contemporáneos.

3. Propuestas de mejora. En base a la experiencia obtenida con el desarrollo de la práctica y con la evaluación y análisis de resultados.

\section{Resultados}

El empowerment del alumnado se empleó como herramienta metodológica para la selección del espacio para efectuar el baño de realidad en el curso 2018-19, obteniendo una gran aceptación por parte del alumnado, quien lo manifestó en repetidas ocasiones, al sentirse protagonista de su propio aprendizaje. El 100\% del alumnado valoró de manera positiva la experiencia del baño de realidad (Figura 6) y, con ello, de extrapolar la docencia a otros espacios formativos.
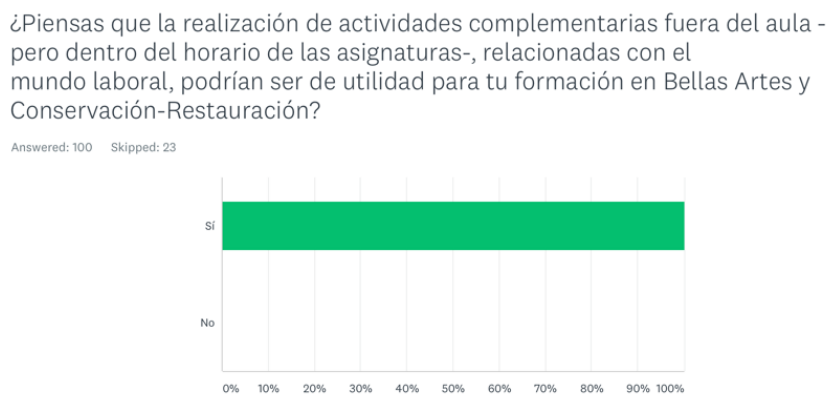

Figura 6. Encuesta con SurveyMonkey®. Fuente: elaboración propia. 
Durante este curso, el baño de realidad se efectuó en el Institut Valencià de Conservació i Restauració de Béns Culturals (IVC+R), el primero electo en la lista de los propuestos por el profesorado, y votado por el alumnado con un $83 \%$ de los votos. En él verificaron procesos de la realidad laboral mediante la observación, que había sido valorada previamente con un $41 \%$. Asimismo, los profesionales de la institución, lanzaron preguntas respecto a materiales y técnicas, estado de conservación de las obras y propuestas de intervención, evaluadas previamente con un $68 \%$ y un $62 \%$ respectivamente, que ellos deliberaban, analizaban y respondían de la manera más rápida posible.

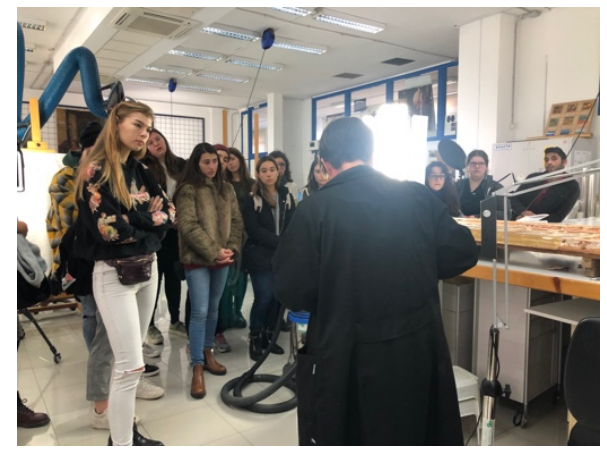

Figura 7. Alumnos en Institut Valencià de Conservació i Restauració de Béns Culturals (IVC+R). Fuente: elaboración propia.

Posteriormente, los mismos profesionales realizaban la resolución del problema planteado, que era recibido por el alumnado con gran motivación, generando dudas que iban resolviendo en el momento. El incremento en la motivación y de la implicación en la práctica fue valorado por medio del portafolio recogido de la actividad.

Asimismo, resulta destacable la implicación por parte del alumnado en la actividad y en las preguntas efectuadas con posterioridad. Por otro lado, la visión profesional ayuda en la autoevaluación del alumnado, con criterios objetivos.

\section{Conclusiones}

La mejora fundamental del empowerment es que el alumnado se siente parte de su propio aprendizaje, desarrollando competencias específicas valiosas, en la selección de actividades y espacios encaminados a su futuro laboral. La percepción por parte del alumnado, con respecto a los baños de realidad siempre resulta positiva y motivadora, dado que entienden que los procesos desarrollados en el aula están encaminados a la resolución de problemáticas reales, con los que se encontrarán al salir al mercado laboral.

Asimismo, la diversificación de espacios, en caso de tener tiempo suficiente con los ECTS asignados a cada asignatura, podría presentar visiones diferentes de una misma profesión. En el contexto de las Bellas Artes y de la Conservación y Restauración de Bienes Culturales se da un factor determinante para la motivación del alumnado, debido al amplio abanico de tipologías de posibilidades laborales futuras, con ello al profesorado le resultará de igual utilidad, puesto que ahondará en las expectativas que el alumnado vuelca cuando se matricula en una asignatura o en un determinado grado o master. 
La metodología docente que se presenta, no obstante, es transferible a cualquier disciplina universitaria, por ello se ha hecho especial hincapié en la difusión de los resultados de la experiencia en medios especializados, para el profesorado interesado en implementar la experiencia con posterioridad.

\section{Agradecimientos}

Al Institut Valencià de Conservació i Restauració de Béns Culturals (IVC+R), por su generosidad en su colaboración, aportando su punto de vista profesional, que fue imprescindible para la elaboración de la presente actividad de baños de realidad, que resultó muy motivador para el alumnado.

Al EICE “AFA-Realidad El proceso de Enseñanza y Aprendizaje Fuera de las Aulas - Baños de Realidad", del Instituto de Ciencias de la Educación de la Universitat Politècnica de València, por brindarnos un espacio en el que poder desarrollar metodologías activas e innovadoras.

\section{Referencias}

CARABAL MONTAGUD, M.A. (2018). “'Reality Baths' as a pedagogical strategy in university education" en 12th annual International Technology, Education and Development Conference. Valencia. 2020-2027.

ESTEBAN GONZÁLEZ, L., ORTEGA GUTIÉRREZ, J. (2017). "El debate como herramienta de aprendizaje" en VIII Jornada de Innovación e Investigación Docente 2017. Universidad de Sevilla. 48-56.

GARCÍA DE PILO, L., USECHE, M.C., SCHLESINGER, M.W. (2014) “Empowerment y compromiso organizacional del personal docente investigador en el ámbito universitario zuliano" en REDHECS. Octubre 2013- Marzo 2014. ISSN: 1856-9331 nº 16.

GAVILAN, D., MARTÍNEZ NAVARRO, G., \& FERNANDEZ LORES, S. (2017). Universitarios y redes sociales informativas: Escépticos totales, moderados duales o prodigitales.

OSPINA RODRÍGUEZ, J. (2006). "La motivación, motor del aprendizaje” en Revista Ciencias de la Salud, octubre, vol. 4, número Especial. Colombia. 158- 160.

TERÁN CAZARES, M.M. (2009). "El empowerment docente: pieza clave para el éxito en el desempeño educativo" en Vincluatégica Efan.

TORRES, A. (2009). "La educación para el empoderamiento y sus desafíos" en Sapiens, Revista Universitaria de Investigación, Año 10. 89. 